\title{
Experience with dedicated geriatric surgical consult services: Meeting the need for surgery in the frail elderly
}

\author{
Rosemarie E Hardin' \\ Thierry Le Jemtel ${ }^{2}$ \\ Michael E Zenilman' \\ 'Department of Surgery, SUNY \\ Downstate Medical Center, Brooklyn, \\ NY, USA; ${ }^{2}$ Department of Medicine, \\ Tulane Medical Center, New Orleans, \\ LA, USA
}

Background: Surgeons are increasingly faced with consultation for intervention in residents of geriatric centers or in patients who suffer from end stage medical disease. We review our experience with consult services dedicated to the needs of these frail patients.

Study design: Patients were prospectively followed after being evaluated by three different geriatric surgical consult services: Group 1 was based at a geriatric center associated with a tertiary medical center, Group 2 was based at a community geriatric center, and Group 3 was based with an hospital-based service for ambulatory patients with end stage congestive heart failure.

Results: A total of 256 frail elderly patients underwent of 311 general surgical procedures ranging from major abdominal and vascular procedures to minor procedures such as debridement of decubitus ulcers, long-term intravenous access, enterostomy and enteral tube placement. Almost half of the surgical volume in Group 1 and 3 were 'maintenance' (decubitus debridement, long term intravenous or stomal or tube care); all of Group 2 were for treatment of decubiti. There was minimal morbidity and mortality from surgery itself, and overall one year survival for Groups 1, 2, and 3 was $46 \%, 60 \%$, and 79\%, respectively. Multivariate analysis showed that each group had its own unique indicators of decreased survival: Group 1 dementia and coronary artery disease, in Group 2 gender and coronary artery disease, and Group 3, gender alone. Age, number of comorbid illnesses, and type of surgery (major vs minor) were not significant indicators.

Conclusions: This is the first review of the role of dedicated surgical consult services which focused on residents of geriatric centers and frail elderly. Conditions routinely encountered in this population such as dementia, end stage disease, multiple comorbidities, polypharmacy, decreased functional and nutritional status are not frequently encountered by general surgeons. But the surgery is safe, and survival data is comparable to those in geriatric centers who did not undergo surgery. A multidisciplinary team approach gives the most effective care, with a primary goal of palliation.

Keywords: surgery in the elderly, geriatrics, surgical consultations, nursing home residents, congestive heart failure, frailty, palliative care, dementia, coronary artery disease, survival

\section{Introduction}

As our population continues to age, elderly patients will develop surgical disease. While much has been published on results of surgical procedures performed on elderly patients, these reports usually conclude that the "healthy" elderly, with good physiologic reserve, can survive to lead functional lives. ${ }^{1}$ Typically, age itself is not a predictor of survival-active comorbid illnesses and emergency surgeries are much more powerful indicators.

Not much is known about the surgical outcomes in the frail elderly. In general, frailty has been defined as "a state of reduced physiologic reserve associated with 
increased susceptibility to disability". ${ }^{2}$ While criteria are being developed to stratify frailty, ${ }^{3}$ in this paper we define frailty as patients with severe medical disability- residents of geriatric centers or patients with end stage medical disease. Almost all of these patients have significantly diminished physiologic reserves, poor nutritional status, significant comorbidities, and mental and physical disabilities.

The general surgeon must be aware of the issues present in this population to provide appropriate care. These include unfamiliar primary diagnoses (dementia, inability to perform basic activities of daily living, end stage congestive heart failure), and operations with palliative, not curative, goals.

The purpose of this paper is to update and review our experience in establishing three different dedicated geriatric consult services. We originally reported our experience with nursing home patients associated with an academic medical center. ${ }^{4}$ We now review this experience and add data from patients we treated at a community based nursing home, and from a unique experience with a frail population of patients cared for by a dedicated congestive heart failure service. We report the patient demographics of a these populations, the type and severity of surgical diseases encountered, procedures peformed, and overall survival after consultation. We also analyze independent variables such as age, gender, presence of coronary artery disease (CAD), significant comorbidity, and their effect on survival within this population.

\section{Methods}

Data was prospectively obtained from three separate cohort studies examining the utility of a general surgical consult service dedicated to providing comprehensive care to elderly and frail patients.

The first cohort (Group 1) has been previously published ${ }^{4}$ and consisted of residents in a geriatric nursing home closely associated with an academic medical center who were referred for general surgical intervention. These patients were followed longitudinally from 1991-1994. Detailed demographic information was obtained including admission diagnosis, consult diagnosis, comorbidities and medications. Information was obtained regarding surgical interventions, and death or discharge from the geriatric facility.

The second cohort of patients (Group 2) consisted of patients residing in a community nursing home that were referred to a surgical consult service established at Albert Einstein College of Medicine from 1995-1997. The bulk of these patients were referred for decubitus care. These patients were prospectively studied and overall survival was analyzed as well as the effect of independent variables such as age, gender, presence of CAD, presence of dementia, and location and severity of ulcer on survival.

The third cohort of patients (Group 3) consisted of ambulatory patients who were followed by the Albert Einstein College of Medicine Heart Failure Group who were referred for elective general surgery. These patients represent a particular subset of the geriatric population since they have a significant comorbidity (congestive heart failure $[\mathrm{CHF}]$ ), and represent an ambulatory population who are frail. These patients were followed prospectively from May 1995 to June 2000. Patient information was obtained regarding demographics, New York Heart Association (NYHA) functional class, comorbid conditions, CHF medications, ejection fraction and social history. Comorbid conditions were defined as significant illness in other organ systems aside from cardiac dysfunction. The endpoints of this study were either death or the termination date of the follow-up period.

For survival statistics, data was entered into a computer database for analysis (Statistica, Statsoft, Tulsa, OK). Survival analysis was first determined for individual groups by Kaplan-Meier product limit estimation. Within each group, univariate analysis of variables was performed by the nonparametric log-rank test for censored data. For determination of risk factors influencing survival, multivariate modeling by Cox proportional hazard model was used. Statistical significance was defined as $\mathrm{p}<0.05$.

\section{Results}

\section{Group I:Academic geriatric center patients referred for surgical care}

This cohort was previously published ${ }^{4}$ and gives a picture of the types of patients referred for consultation at geriatric centers associated with academic medical centers.

\section{Demographics}

This cohort consisted of a total of 153 patients, with the mean age of $72.9 \pm 1.1$ years, and followed for an average of $14.5 \pm 0.6$ months (range 0-24 months). A total of 168 procedures were performed in 117 patients; the distribution of types is depicted in Table 1 (Group 1). Polypharmacy was observed in most of the patients; only $30 \%$ were on no medications.

\section{Survival}

The 30-day mortality rate following all surgical interventions was $8.5 \%$. Using Kaplan-Meier product limit estimations, the one-year actuarial survival rate for this cohort was $46 \%$ and the two-year survival rate was $33 \%$ (Figure 1). Multivariate 
Table I Comparison of operative procedures done in patients referred to geriatric surgical consultation services

\begin{tabular}{ll}
\hline Group I: Distribution of I68 operative procedures performed in Group I \\
\hline Procedure, specific & Percent \\
Decubitus, ulcer debridement & 25 \\
Amputations (all types) & 13 \\
Intravenous Access (all types) & 15 \\
Breast: & 8 \\
Biliary & 7 \\
Endoscopy or placement of gastrostomy tube & 7 \\
Laparotomy & 16 \\
Hernia & 3 \\
Other* & 6 \\
\hline
\end{tabular}

Notes: *other = parathyroid, tracheostomy, lymph node biopsy and anorectal procedure.

Group 3 Distribution of 38 operative procedures performed in Group 3

\begin{tabular}{ll}
\hline Operations & Percent \\
\hline Long term intravenous for inotrope & 29 \\
Colectomy/bowel resection & 24 \\
Lap-cholecystectomy/Lap Nissen & 14 \\
Rectal Surgery/Hemorrhoidectomy & 16 \\
Jejunostomy & 3 \\
Percutaneous cholecystostomy & 3 \\
Vein excision & 5 \\
Hernia repair & 5 \\
Open drainage of psoas abscess & 3 \\
\hline
\end{tabular}

Notes: Group I data adapted from Zenilman ME, Bender JS, Magnuson TH, Smith GS. General surgical disease in the nursing home patient: Results of a dedicated geriatric surgery consult service. JAm Coll Surg. 1996;|83:36I-370. All the operations in Group 2 were bedside decubitus debridements.

factors that affected survival are depicted in Table 2a). There was no difference in survival when patients were stratified by gender, by major or minor surgery, or by the presence of an active do not resuscitate order.

\section{Group 2: Community geriatric center patients referred for surgical care}

This cohort gives a picture of the types of patients referred for surgical consultation from community geriatric centers.

\section{Demographics}

There were of 105 nursing home patients referred to the consult service, all for debridement of chronic decubitus ulcers. The mean age of this cohort was $75 \pm 1.3$ year. Seventy percent were female, a higher percentage compared to Group 1. These patients were followed from January 1995 to August 1997 for a mean \pm SEM of $10.9 \pm 1.0$ months (range 0-36 months). Thirty-four percent suffered from CAD, 31\% had diabetes, $21 \%$ respiratory disease, and $9 \%$ from renal disease.

Most decubiti were debrided at the bedside in the nursing facility. Thirty-three percent were extremity decubiti, 27\% trunk, and $17 \%$ were on both the extremity and trunk, and $23 \%$ were not specified.

\section{Survival}

Most surgeries in this group were minor. We observed oneand two-year actuarial survival rates to be $60 \%$ and $42.7 \%$, respectively (Figure 1). Univariate analysis demonstrated that in this population, gender and the presence of coronary artery disease significantly affected overall survival, but age greater than 70, presence of dementia or comorbid illness did not. Multivariate analysis confirmed this (Table 2b).

\section{Group 3: Heart failure patients referred for elective general surgery}

This cohort gives a picture of the types of patients referred from specialized hospital-based consult services of ambulatory frail patients.

\section{Demographics}

This cohort consisted of 45 patients referred to surgery from a population of 600 patients with severe CHF managed by a dedicated service. Their average age was 65.3 years (median 67) and 38\% were women. The average NYHA class of CHF at first enrollment in cohort was $2.9 \pm 0.1$, at the time of surgical intervention was $3.34 \pm 0.12$, and at final follow up for survivors was $3.16 \pm 0.12$. The mean Goldman score ${ }^{5}$ for this cohort was $25.1 \pm 2.1$. At the time of surgical intervention the mean left ventricular ejection fraction was $29.6 \pm 1.7 \%$. Patients were followed from May 1995-June 2000, for a mean \pm SEM $16.3 \pm 2.2$ months (range 0-64 months).

Seventy seven percent of this cohort had additional significant comorbid conditions including diabetes, hypertension or renal insufficiency. As we observed in the academic geriatric center patients (Group 1) polypharmacy was common, but in this group the medications were focused on the primary cardiac disease. Most patients (70\%) were on an angiotensin-converting enzyme (ACE) inhibitor and diuretic, while $35 \%$ were on triple therapy: an ACE inhibitor, digoxin, and diuretic. Ten to thirty percent were on calcium channel blockers, $\beta$-blockers, or antiarrythmics.

Thirty-four patients in this group underwent 38 procedures. Fifteen major abdominal procedures were performed in this cohort (Table 1). These included eight 
Comparative Survival of Services

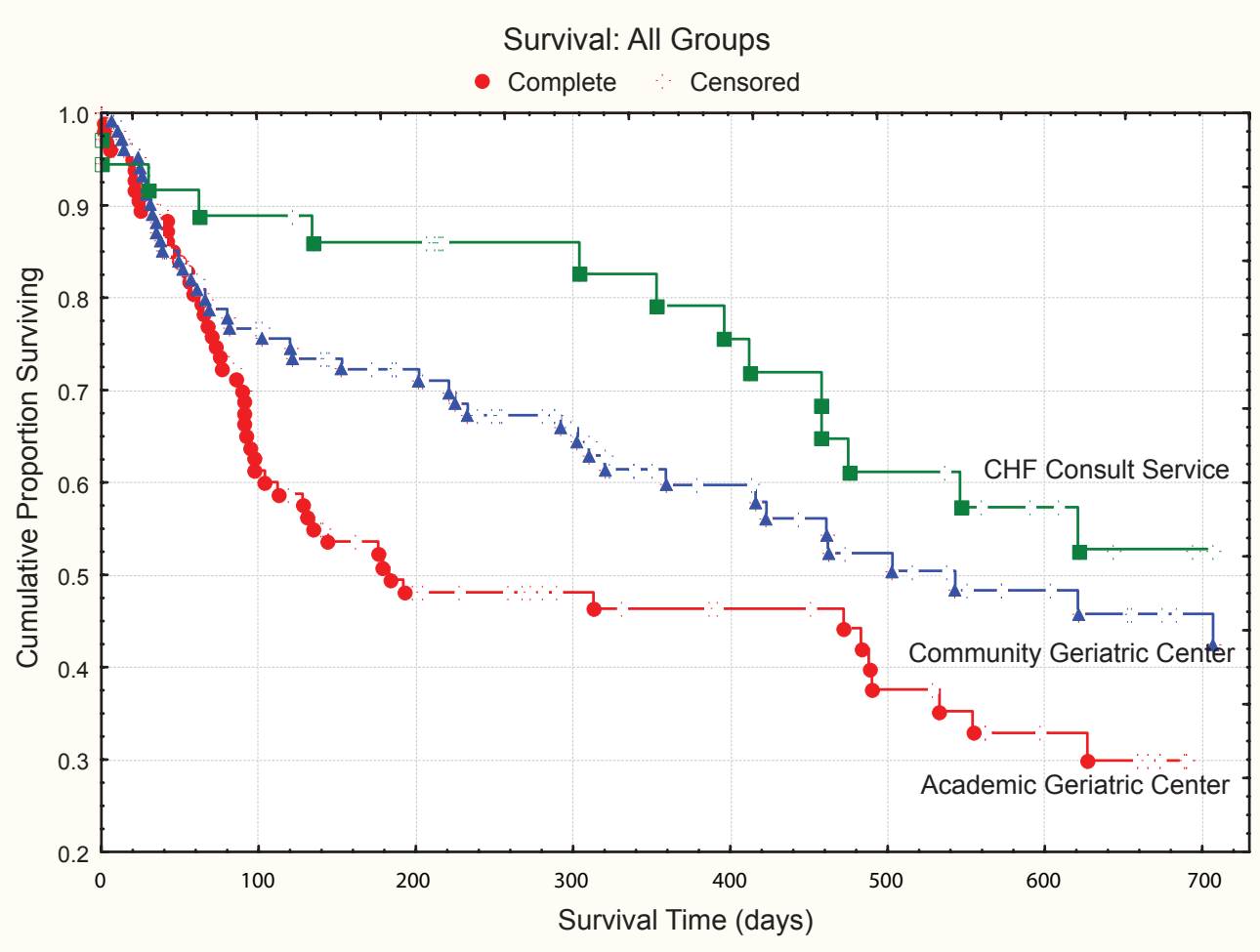

267 patients, 311 operations

Figure I Overall survival for the three groups observed. Data for "academic geriatric center" is adapted from Zenilman and Colleagues (I996).

colectomies for cancer, one intestinal resection for chronic gastrointestinal bleed, open drainage of a psoas abscess, laparoscopic cholecystectomy and one laparoscopic Nissen fundoplication for gastrointestinal reflux. In this group there were three complications (20\%) and one postoperative death $(6.7 \%)$. The latter was in a patient who underwent uneventful right colectomy for cancer and suffered a fatal complication from a balloon thermodilution catheter on postoperative day 1 .

Twenty-three procedures were performed under local anesthesia, the most common being 'maintenance' surgerycentral venous access for inotropic support (Table 1). Other procedures performed under local anesthesia included transanal excision of a rectal cancer, hemorrhoidectomy, feeding jejeunostomy, and a vein excision for thrombophlebitis. There were two complications in this group $(9.1 \%)$ and no deaths.

\section{Survival}

One- and two-year survival for the entire group was $78.5 \%$ and $48.8 \%$, respectively (Figure 1). Univariate analysis showed that age greater than 65 years, male gender, the presence of any other comorbid illness, use of tobacco or ethanol and race adversely affected survival (Table 2c). Multivariate analysis of risk revealed that of all the factors, only female gender was the factor predictive of increased survival.

Importantly, as we observed in Group 1, when patients in this group underwent major abdominal surgery with general anesthesia were compared to those who had local procedures (predominantly chronic central venous catheters placed for home inotropic therapy), survival rates were similar.

\section{Discussion}

This report updates our experience in establishing dedicated geriatric surgical consult services to provide care for the frail elderly: a rapidly growing segment of the general surgical population. A total of 311 procedures performed in 267 patients is reported; the largest to date. The study has three potential flaws. First, there is a selection bias since the patients, while frail, were healthy enough to be referred for elective surgery. This is a potential strength since by not addressing acute illnesses we were able to give a more accurate picture of nonemergency surgery in the frail. Second, the three groups studied seemed very different. 
Table 2 Comparison of multivariate analysis of variables affecting survival

\begin{tabular}{|c|c|c|}
\hline \multicolumn{3}{|l|}{ A. Group I } \\
\hline Variable & Relative risk & $\mathbf{p}$ \\
\hline CAD & 3.27 & 0.01 \\
\hline MMS $<24$ & 2.39 & 0.04 \\
\hline Age $>70$ & 2.03 & 0.06 \\
\hline$<I$ month in GC & 1.72 & $>0.1$ \\
\hline \# medications & 0.98 & $>0.1$ \\
\hline \# diseases & 0.86 & $>0.1$ \\
\hline \multicolumn{3}{|l|}{ B. Group 2} \\
\hline Male & 2.83 & 0.004 \\
\hline CAD & 2.20 & 0.008 \\
\hline Age $>70$ & 1.47 & 0.33 \\
\hline Dementia & 1.36 & 0.31 \\
\hline$>$ lyr in GC & 1.04 & 0.91 \\
\hline \multicolumn{3}{|l|}{ C. Group 3} \\
\hline Male & 3.67 & 0.02 \\
\hline Any comorbidity & 1.71 & 0.47 \\
\hline Use of tobacco/ & 1.71 & 0.46 \\
\hline \multicolumn{3}{|l|}{ ETOH } \\
\hline$>65$ years & 1.19 & 0.80 \\
\hline White vs black & 1.14 & 0.80 \\
\hline ASA Class & 0.891 & 0.84 \\
\hline CHF meds & 0.798 & 0.56 \\
\hline
\end{tabular}

Notes: Zenilman ME, Bender JS, Magnuson TH, Smith GS. General surgical disease in the nursing home patient: Results of a dedicated geriatric surgery consult service.J Am Coll Surg. 1996; 183:36I-370.

Abbreviations: $\mathrm{ACE}$, angiotensin-converting enzyme; $\mathrm{ASA}$, Anesthesia Class $=1$, 2, 3, 4; Any comorbidity, any other than CHF; CAD, coronary artery disease; $\mathrm{CHF}$, congestive heart failure; CHF meds, number of drugs (ACE inhibitors/diuretic/lasix) taken (stratified I, 2,3) GC, geriatric center; MMS, Mini Mental Status;Tobacco-ETOH, chronic use of either tobacco or ethanol.

While their venues did differ, from a geriatric perspective, all the patients were frail elderly.

\section{Observations}

The important observation was that common general surgical disease occurs in patients who reside in geriatric centers and who have end stage medical disease (Table 1). While 'maintenance care' (drainage/feeding tubes, decubitus care, chronic intravenous access) made up a significant proportion of the procedures $(>45 \%$ in Group $1 ; 100 \%$ in Group 2; $32 \%$ in group 3 ), other common surgical procedures such as biliary, abdominal, hernia, breast surgery were also performed, with minimal morbidity and mortality.

The observed survival for patients in these groups was poor. For patients who were residents in academic and community geriatric centers the one year survival was $50 \%-60 \%$. For those outpatients with controlled, but significant CHF, survival was better at nearly $80 \%$. This difference is likely attributed to the differences in frailty of the populations. Specifically, the institutionalized patients have less mobility, more dementia, and more chronic conditions necessitating 'maintenance care'. The patients with end stage CHF had comorbid illnesses, but they had no dementia and were outpatients.

Univariate analysis in each group showed that multiple factors contributed to survival, such as age, dementia, gender, $\mathrm{CAD}$, length of stay in the geriatric center, number of comorbidities, polypharmacy, and even use of tobacco or ethanol in patients with CHF. When subjected to the more rigorous multivariate analysis, most variables - including age - fell out of statistical significance (Table 2), a phenomenon seen in many other geriatric studies. In the academic geriatric center, coronary artery disease, and dementia were significant predictors of survival: gender was not even significant using univariate analysis. ${ }^{4}$ But, in the community geriatric center and CHF outpatient group, gender and CAD was a predictor. The type of surgery (major vs minor) or the presence of a do-not-resusicate (DNR) order had no impact on life expectancy. The small group size of the CHF group (Group 3) does preclude accurate statistical analysis, but the fact remains that these frail patients did do well after major elective surgery (only one death out of 15).

\section{Issues for surgeons who take care of these patients}

\section{Geriatric centers}

In 1999 there were 2.5 million discharges from geriatric centers in the US. ${ }^{6}$ Ninety percent of these residents were over the age of 65 . Approximately $37 \%$ of these residents were over the age of 85 . Kemper and colleagues showed that the expected use of geriatric centers is high: $36 \%, 60 \%$, and $71 \%$ for patients grouped 75-84 years, 85-94 years, and greater than 95 years, respectively. It is expected that $43 \%$ of persons over 60 years of age will eventually use a nursing home. Thirty-two percent will reside there for over three months, $24 \%$ for over one year, and $9 \%$ for over five years. ${ }^{7}$

Figure 2 demonstrates the current demographic profile of US geriatric center residents. Frailty is common in this group. Nearly half of these residents are admitted from a medical facility and one of the most common causes for discharge from a nursing facility was for admission to a hospital. A significant proportion of this elderly population will therefore require medical care, some of which will be surgical. A specialized geriatric general consult service for these frail patients would 


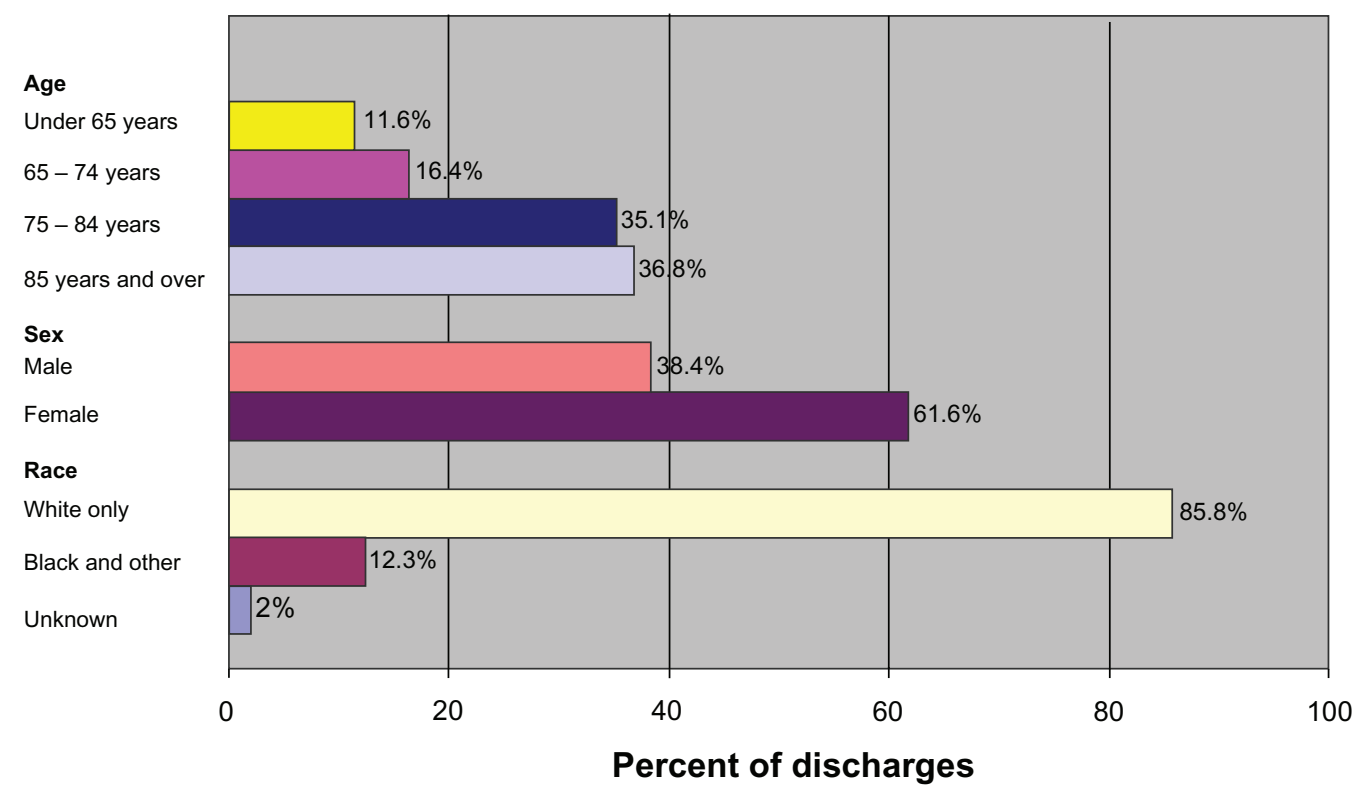

Percent distribution of discharges by age, sex, and race: United States, 1998 - 99

Figure 2 Current demographic profile of US geriatric center residents. Adapted from Jones A.The National Nursing Home Survey: 1999 Summary. National Center for Health Statistics. Vital Health Stat. I3(I52)2002: I-I I6.

provide a forum to coordinate for both maintenance care and major abdominal and vascular procedures.

\section{Frailty}

Frailty is a chronic condition, but can also be the result of an acute change in functional reserve. Criteria for frailty have been established: age greater than 85 years, dependence in one or more activities of daily living, presence of three or more comorbid conditions and presence of one of more geriatric syndromes (such as delirium, incontinence, osteoporosis, falls risk and failure to thrive). ${ }^{7}$ Patients who reside in geriatric centers and outpatients with end stage medical diseases (such as CHF) should also be considered frail.

Frail elderly are encountered in the hospital, geriatric center, and outpatient setting. Understanding the frail elderly can help prevent the progression of disability and reduce the risk of unplanned hospitalizations, nursing home admissions and reductions in cost of medical care delivery to this population. While patients over 65 years of age account for $31 \%$ of acute care hospital admissions and $45 \%$ of hospital expenditures for adults, ${ }^{8,9}$ frail elderly require more medical resources. ${ }^{10}$

The median survival of a frail elderly patient has been estimated to be in excess of two years. Surgical intervention should therefore not be aimed at increasing survival but rather maintain quality of remaining life. ${ }^{11}$ This paradigm is of particular importance to general surgeons who are not all trained under this paradigm.

\section{Congestive heart failure}

The annual incidence of CHF is near 10 per 1,000 (in patients over 65 ), and the prevalence has increased to some 400,000 new cases each year, with at least 4-5 million people affected in the US. ${ }^{12}$ This translates to 875,000 hospitalizations and 2.9 million physician visits per year. In 1993, there were 42,000 reported deaths from CHF, with an additional 219,000 deaths where CHF was a significant contributor.

While the prognosis for patients with CHF is improving, there are no accurate predictors of survival. Studies have shown that CHF patients have a greater than $50 \%$ predicted chance of living two months on the day before their actual death. ${ }^{13-15}$ These are patients now being referred for both minor and major surgery.

Table 3 Tools for comprehensive geriatric assessment that can be employed by dedicated consultation services

\begin{tabular}{ll}
\hline Geriatric variable & Analysis tool \\
\hline Comorbidity & $\begin{array}{l}\text { Comorbidity index (Charlson scale } \\
\text { and the Chronic Illness Rating Scale) } \\
\text { Instrumental Activities of Daily } \\
\text { Lunction }\end{array}$ \\
& Living (IADL) and Activities of Daily \\
Mental Function & Folstein Mini Mental Status (MMS) \\
& and Assessment for Dementia \\
& Dementia Rating Scale (DRS) \\
Malnutrition & Mini Nutritional Assessment \\
\hline
\end{tabular}




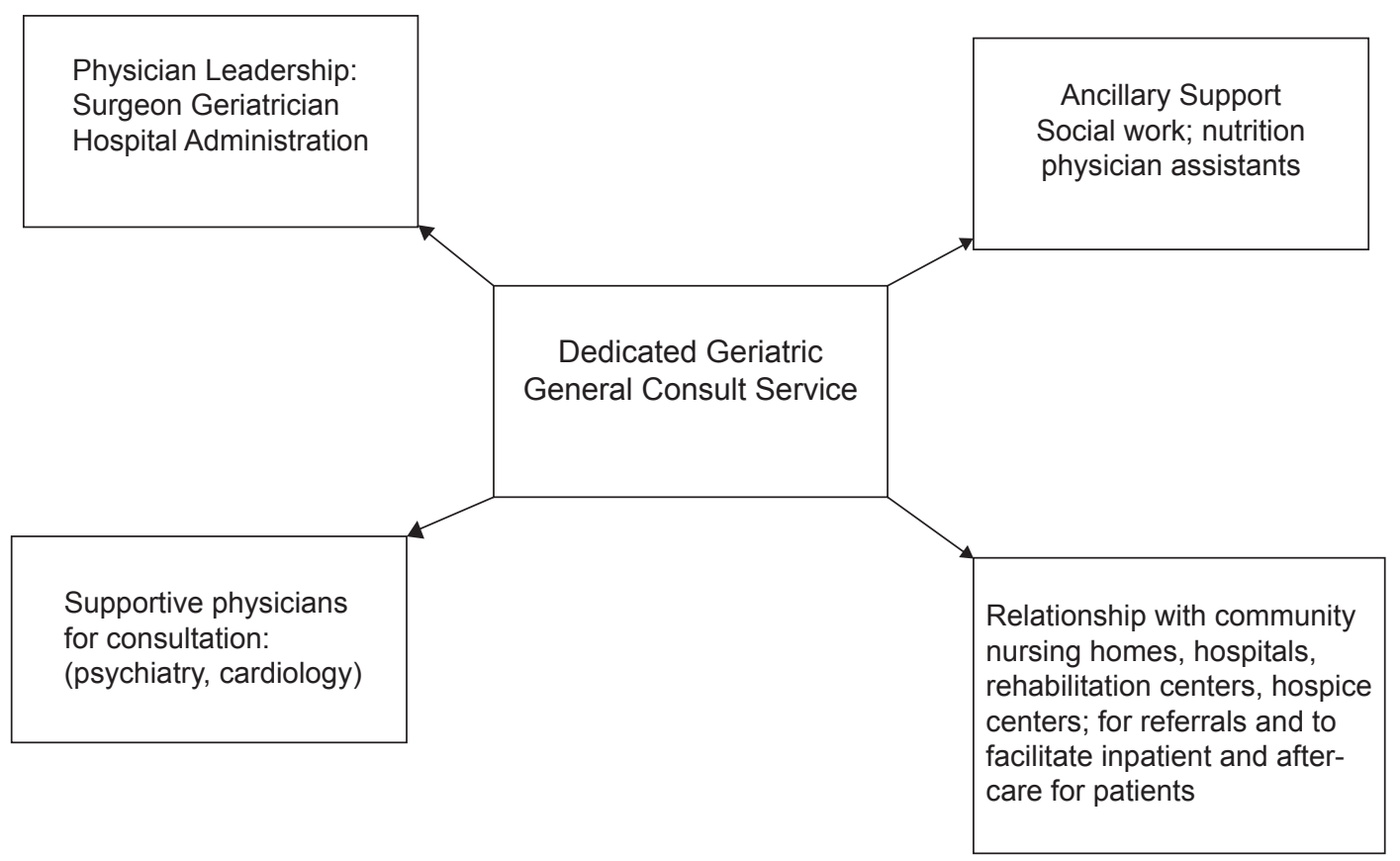

Figure 3 The multidisciplinary approach to the geriatric patient consult service.

\section{The case for focused geriatric surgery consult services}

Frail and geriatric patients present with medical issues that general surgeons are not trained to recognize or treat: dementia, delirium, depression, propensity for falls, multiple medical comorbidities and polypharmacy, depressed cognition and nutrition, and problems with social support, alcohol and abuse. ${ }^{14}$ The need for a dedicated multidisciplinary team approach to address these is critical (Figure 3).

The earliest trial evaluating the efficacy of a hospital based geriatric evaluation unit was conducted in 1979 at the Sepulveda VA Medical Center. ${ }^{16}$ Elderly patients who were assigned to the unit showed a $50 \%$ reduction in one year mortality compared with the control group, and less need for long-term care. Furthermore, the study demonstrated improved diagnostic accuracy, reduced use of medications, reduced need for institutionalization and improved functional status.

Several other clinical trials evaluating geriatric evaluation services yielded with conflicting results on survival and cost effectiveness. ${ }^{17}$ But they generally concluded that geriatric units have a positive impact on elderly patients' quality of life, examples being in "nonmedical" interventions such as cognition, depression or preventative measures. ${ }^{18,19}$ At the very least, a dedicated unit will efficiently implement the necessary medical, social, and economic interventions, all of which can be complicated.

\section{Elements of the service}

A multidisciplinary, integrated team can perform a comprehensive geriatric assessment that will be able to guide appropriate management, for the frail geriatric patients (Figure 3). A comprehensive nonsurgical geriatric assessment includes overall health status, functional status, nutrition, cognition, socioeconomic and emotional evaluations, assessment for geriatric syndromes such as delirium, incontinence, osteoporosis, risk of fall and failure to thrive..$^{18}$ Once completely assessed, the role for surgery, whether curative, palliative, or maintenance, can be decided.

For this team to function, a physician leader, usually a surgeon and geriatrician, and support staff, nurses, social workers, nutritionists, psychologists, and physician extenders, are all needed. A dedicated unit with appropriate physical facilities, eg, support railings in the hallway, handicapped baths, day-rooms, signs for daily orientation, is essential.

\section{Disclosure}

The authors report no conflicts of interest in this work.

\section{References}

1. Pofahl WE, Pories WJ. Current status and future directions of geriatric general surgery. J Am Geriatr Soc. 2003;51:S351-S354.

2. Buchner DM, Wagner EH. Preventing frail health. Clin Geriatr Med, 1992;8:1-17.

3. Ferrucci L. The frailty syndrome: a critical issue in geriatric oncology. Crit Rev Oncol Hermatol. 2003;46:127-137. 
4. Zenilman ME, Bender JS, Magnuson TH, Smith GS. General surgical disease in the nursing home patient: Results of a dedicated geriatric surgery consult service. J Am Coll Surg. 1996;183:361-370.

5. Goldman L, Caldera DL, Nussbaum SR, et al. Multifactorial index of cardiac risk in noncardiac surgical procedures. $N$ Engl J Med. 1977;297:845-850.

6. Jones A. The National Nursing Home Survey: 1999 Summary. National Center for Health Statistics. Vital Health Stat. 2002;13(152):1-116.

7. Kemper P, Murtaugh CM. Lifetime use of nursing home care. $N$ Engl J Med. 1991;324:595-600.

8. Winograd CH, Gerety MB, Brown E, Kolodny V. Targeting the hospitalized elderly for geriatric consultation. J Am Geriatr Soc. 1988;36:1113-1119.

9. Landefeld CS, Palmer RM, Kresevic DM, Fortinsky RH, Kowal J. A randomized trial of care in a hospital medical unit especially designed to improve the functional outcomes of acutely ill older adults. $N \mathrm{Engl}$ J Med. 1995;332:1338-1344.

10. Ferrucci L, Guralnik JM, Cavazzini C, et al. The frailty syndrome: a critical issue in geriatric oncology. Crit Rev Oncol Hematol. 2003;46:127-137.

11. Repetto L, Venturino A, Fratino L, et al. Geriatric oncology: a clinical approach to the older patient with cancer. Eur J Cancer. 2003;39:870-880.
12. Eichorn EJ. Prognosis determination in heart failure. Am J Med. 2001;110:14-35.

13. Levenson JW, McCarthy EP, Lynn J, et al. The last six months of life for patients with congestive heart failure. J Am Geriatr Soc. 2000;48(5 Supp1):S101-S109.

14. Teno JM, Harrell FE, Knaus W, et al. Prediction of survival for older hospitalized patients: The HELP survival model. J Am Geriatr Soc. 2000;48(5 Suppl):S516-S524.

15. Balducci L, Beghe C. Cancer and age in the USA. Crit Rev Oncol Hemotol. 2001;37:137-145.

16. Rubenstein LZ, Wieland D, English P, Josephson K, Sayre JA, Abrass IB. The Sepulveda VA Geriatric Evaluation Unit: data on four-year outcomes and predictors of improved patient outcomes. $J \mathrm{Am}$ Geriatr Soc. 1984;32:503-512.

17. Ellis G, Langhorne P. Comprehensive geriatric assessment for older hospital patients. Br Med Bull. 2005;71:45-59.

18. Balducci L, Beghe $\mathrm{C}$. The application of the principles of geriatrics to the management of the older person with cancer. Crit Rev Oncol Hematol. 2000;35:147-154.

19. Reuben DB, Borok GM, Wolde-Tsadik G, et al. Randomized trial of comprehensive geriatric assessment in the care of hospitalized patients. N Engl J Med. 1995;332:1345-1350. 